\title{
Manno-epi-cyclophellitols Enable Activity-Based Protein Profiling of Human $\alpha$-Mannosidases and Discovery of New Golgi Mannosidase II Inhibitors
}

\author{
Zachary Armstrong, Chi-Lin Kuo, Daniël Lahav, Bing Liu, Rachel Johnson, Thomas J. M. Beenakker, \\ Casper de Boer, Chung-Sing Wong, Erwin R. van Rijssel, Marjoke F. Debets, Bogdan I. Florea, \\ Colin Hissink, Rolf G. Boot, Paul P. Geurink, Huib Ovaa, Mario van der Stelt, Gijsbert M. van der Marel, \\ Jeroen D. C. Codée, Johannes M. F. G. Aerts, Liang Wu, Herman S. Overkleeft,** and Gideon J. Davies*
}

Cite This: J. Am. Chem. Soc. 2020, 142, 13021-13029

Read Online

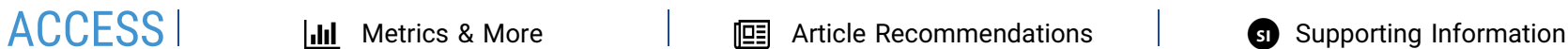

ABSTRACT: Golgi mannosidase II (GMII) catalyzes the sequential hydrolysis of two mannosyl residues from GlcNAc$\mathrm{Man}_{5} \mathrm{GlcNAc}_{2}$ to produce GlcNAcMan $\mathrm{GlcNAc}_{2}$, the precursor for all complex $\mathrm{N}$-glycans, including the branched $\mathrm{N}$-glycans associated with cancer. Inhibitors of GMII are potential cancer therapeutics, but their usefulness is limited by off-target effects, which produce $\alpha$-mannosidosis-like symptoms. Despite many structural and mechanistic studies of GMII, we still lack a potent and selective inhibitor of this enzyme. Here, we synthesized manno-epicyclophellitol epoxide and aziridines and demonstrate their covalent modification and time-dependent inhibition of GMII.

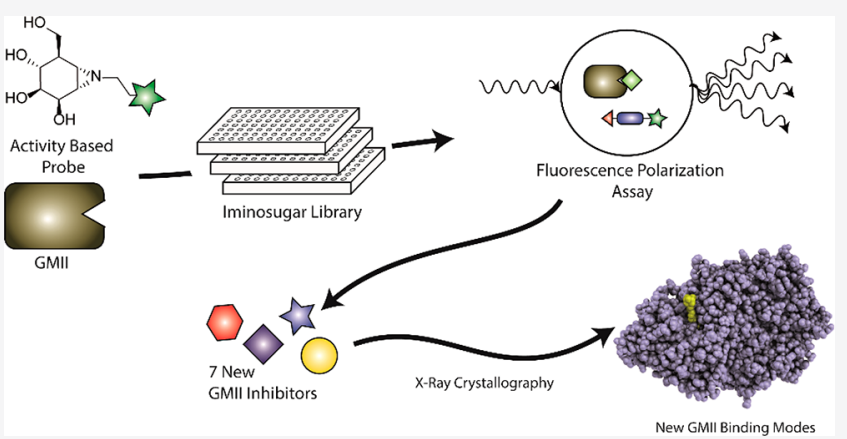
Application of fluorescent manno-epi-cyclophellitol aziridine derivatives enabled activity-based protein profiling of $\alpha$-mannosidases from both human cell lysate and mouse tissue extracts. Synthesized probes also facilitated a fluorescence polarization-based screen for dGMII inhibitors. We identified seven previously unknown inhibitors of GMII from a library of over 350 iminosugars and investigated their binding modalities through X-ray crystallography. Our results reveal previously unobserved inhibitor binding modes and promising scaffolds for the generation of selective GMII inhibitors.

\section{INTRODUCTION}

Golgi $\alpha$-mannosidase (GMII, MAN2A1, mannosyl-oligosaccharide 1,3/1,6- $\alpha$-mannosidase) is a glycoside hydrolase from $\mathrm{CAZy}$ family $\mathrm{GH} 38^{1}$ that catalyzes sequential hydrolysis of both the terminal $\alpha$-1,3- and 1,6-linked mannoses from the $N$ glycan GlcNAcMan GlcNAc $_{2}$ (Figure 1A), which is a committed step in the formation of complex $\mathrm{N}$-glycans. ${ }^{2}$ Altered $\mathrm{N}$-glycan branching has been directly linked to the metastatic potential of cells, ${ }^{3,4}$ and consequently, inhibitors of GMII are a potential target for anticancer therapeutics. The potent GMII mannosidase inhibitor swainsonine (Figure 1B), first identified in locoweed, ${ }^{5}$ has been investigated in this capacity. Swainsonine treatment reduces metastasis and improves outcome in breast, colon, and skin cancers. ${ }^{6-8}$ However, swainsonine also produces side effects resembling the lysosomal storage disease $\alpha$-mannosidosis ${ }^{9,10}$ resulting from the off-target inhibition of lysosomal $\alpha$-mannosidase MAN2B1 (LAM).

As GMII is an important anticancer target, many studies have sought to identify or design new and selective inhibitors. One avenue that has been explored is the modification of existing GMII inhibitors such as swainsonine and mannostatin A (Figure 1B). Swainsonine derivatives containing C5substituents have been investigated, and although these compounds maintain inhibitory potency against Drosophila GMII (dGMII; 40\% identity to human GMII), selectivity over Drosophila lysosomal mannosidase (dLAM; 45\% identity to human LAM) is modest. ${ }^{11}$ Mannostatin A, a natural product $\alpha$-mannosidase inhibitor produced by Streptoverticillium verticillus $^{12}$ (Figure $1 \mathrm{~B}$ ), has also been derivatized in a similar fashion; ${ }^{13}$ however the analogues had a reduced specificity for human GMII over human LAM. Another class of inhibitor, the hydroxylated pyrrolidines, has also been investigated in the context of GMII inhibition. This class of mannosidase inhibitor was first identified by Popowycz et al., ${ }^{14}$ and functionalized

Received: April 9, 2020

Published: July 1, 2020 


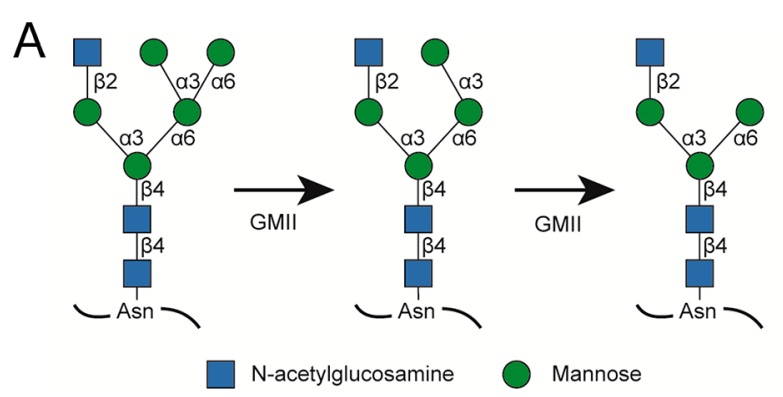

B<smiles>CS[C@H]1[C@@H](N)[C@H](O)[C@@H](O)[C@H]1O[Mg]</smiles><smiles>OC[C@H]1[C@@H](O)[C@H](O)c2nccn21</smiles><smiles>O=C(Cc1ccc(F)cc1)NC[C@H]1N[C@H](CO)[C@@H](O)[C@H]1O</smiles>

Mannoimidazole

Polyhydroxylated Pyrrolidine Derivative

Figure 1. (A) Catalytic activity of Golgi $\alpha$-mannosidase. Historical inhibitors of GMII.

hydroxylated pyrrolidines have subsequently been determined to be more selective toward GMII. ${ }^{15-18}$ A different approach to identify inhibitors was employed by Kuntz and coworkers. ${ }^{19}$ Using a fluorogenic reporter substrate the authors screened a focused library of potential glycosidase inhibitors for action against dGMII. This study revealed several compounds including glucoimidazole and mannoimidazole, both with low micromolar inhibition constants for dGMII.

In the pursuit of new inhibitors of GMII we have applied the considerable power of cyclophellitol-like activity-based probes (ABPs). ${ }^{20}$ Herein we report the covalent inhibition of GMII with manno-epi-cyclophellitol epoxide and aziridine. A fluorescent derivative of the manno-epi-cyclophellitol aziridine enabled activity-based protein profiling (ABPP) of GH38 $\alpha$ mannosidases in both cells overexpressing human GH38s and mouse tissue extracts. This labeling could be reduced with known inhibitors of GH38s. We next developed a highthroughput fluorescence polarization ABPP screen for $\alpha$ mannosidase inhibitors. Screening of our in-house focused library of over 350 iminosugars revealed seven previously unidentified GMII inhibitors, some of which were found by Xray crystallography to bind in modes distinct from previously observed inhibitors. Together this gives us fresh insight into the design of GMII inhibitors and lead compounds for the creation of second-generation GMII inhibitors while also providing a high-throughput screening protocol for future library assessment.

\section{RESULTS AND DISCUSSION}

Manno-epi-cyclophellitols Are Time-Dependent Inhibitors of dGMII. GMII is a GH38 retaining $\alpha$-Dmannosidase that employs a two-step, double-displacement mechanism in which a covalent-enzyme intermediate is flanked by oxocarbenium-ion-like transition states. ${ }^{21}$ We reasoned that as the GH38 mechanism processes through a covalent intermediate, GMII would be an ideal target for the cyclophellitol class of $\mathrm{GH}$ inhibitors, which have become a powerful scaffold for the creation of activity-based glycosidase probes. $^{20}$ The cyclophellitol class of GH inhibitors covalently modify retaining GHs, and activity-based probes bearing cyclophellitol warheads have enabled high-throughput inhibitor and drug discovery, ${ }^{22}$ biomass enzyme profiling, ${ }^{23}$ and detailed conformational and structural insight. ${ }^{24,25}$

Both the manno-epi-cyclophellitol epoxide (1) and aziridine (2) were synthesized (see Figure 2 and the Supporting<smiles></smiles><smiles>CC(=O)NCc1cn(C)nn1</smiles><smiles>OCC1C(O)C(O)C(O)[C@@H]2NC12</smiles>

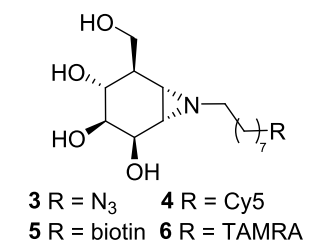

$5 \mathrm{R}=$ biotin $6 \mathrm{R}=$ TAMRA
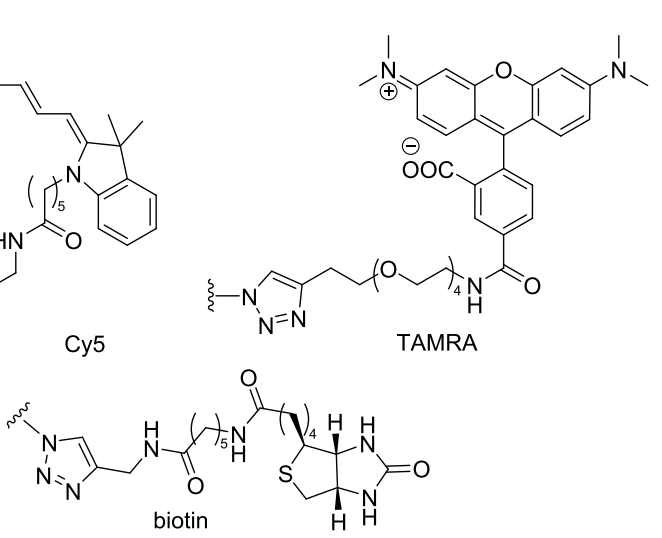

Figure 2. Manno-epi-cyclophellitol inhibitors and probes used in this work.

Information for synthetic details) to ascertain whether these inhibitors could target dGMII. When $\mathbf{1}$ and $\mathbf{2}$ were incubated with recombinant dGMII, we observed that both displayed time-dependent inhibition, which is typical for cyclophellitoltype inhibitors (see Figure S1). To compare the reactivity of these two compounds, second-order rate constants $\left(k_{\text {inact }} / K_{\mathrm{I}}\right)$ were determined using 4-methylumbelliferyl $\alpha$-D-mannopyranoside (MU- $\alpha$-Man) as a substrate. The $k_{\text {inact }} / K_{\mathrm{I}}$ value for $\mathbf{1}$ was determined to be $50 \pm 5 \mathrm{~min}^{-1} \mathrm{M}^{-1}$, while the observed inhibition constant for aziridine 2 was over 100-fold higher $\left(5500 \pm 100 \mathrm{~min}^{-1} \mathrm{M}^{-1}\right)$. This enhanced inhibition of 2 is consistent with the trend previously observed for $\alpha$ glucosidases by epi-cyclophellitol epoxides and aziridines. ${ }^{26}$

We next obtained a crystal structure of dGMII that had been soaked with aziridine 2 . The reacted manno-epi-cyclophellitol moiety is covalently linked to the nucleophile Asp204 in the Xray structure (Figure 3B). This structure clearly shows the opening of the aziridine ring resulting from nucleophilic attack at the pseudoanomeric carbon (Figure 3A). The resulting enzyme-bound cyclitol structure displays a ${ }^{1} S_{5}$ skew boat conformation, which is consistent with the proposed ${ }^{\mathrm{O}} S_{2} / B_{2,5}$ $\rightarrow B_{2,5}{ }^{\ddagger} \rightarrow{ }^{1} S_{5}$ reaction itinerary for GH38 $\alpha$-mannosidases. ${ }^{21,27}$ Comparison to the structure of 2-deoxy-2-fluoro-Dmannose bound to the dGMII D341N acid/base mutant ${ }^{21}$ 

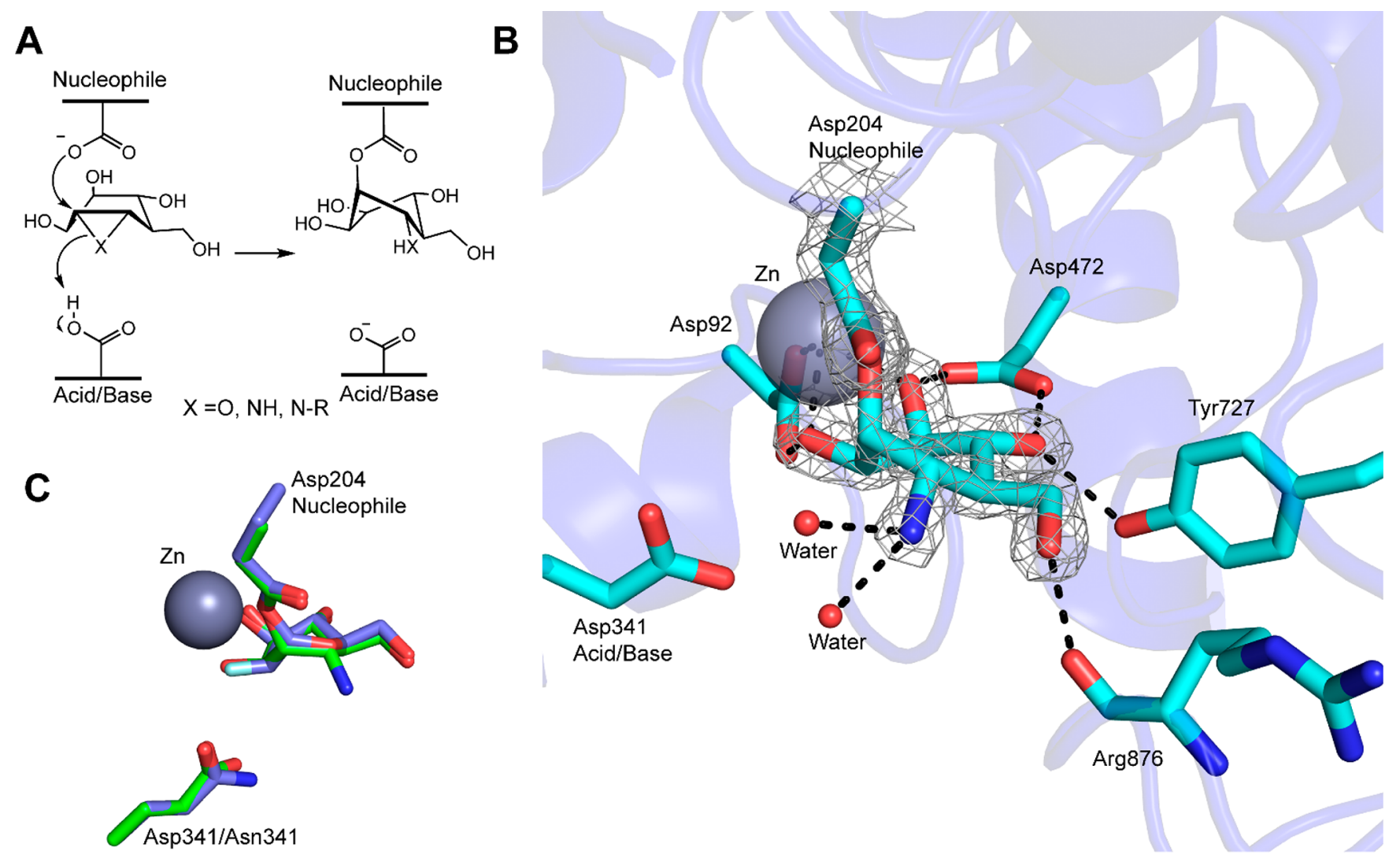

Figure 3. Covalent inhibition of dGMII with manno-epi-cyclophellitol aziridine. (A) Mechanism of covalent inhibition of $\alpha$-mannosidase by manno-epi-cyclophellitols. (B) Structure of 2 covalently linked to dGMII. The $2 F_{\mathrm{o}}-F_{\mathrm{c}}$ map is contoured at $1.5 \sigma,\left(0.48 e^{-} / \AA^{3}\right)$. (C) Overlay of 2 covalently bound to dGMII and 2-deoxy-2-fluoro-D-mannose bound to the D341N variant of dGMII (PDB: 1QX1). The bound (and reacted) manno-epi-cyclophellitol aziridine is shown in green, while the bound 2-fluoromannose is shown in blue.

(PDB ID: 1QX1) revealed excellent concordance with the bound 2-fluorosugar-enzyme intermediate (Figure 3C).

The rapid time-dependent inactivation of dGMII by 2 and the orientation of the amine toward the solvent suggested that this inhibitor could serve as the scaffold for the design of activity-based probes. Toward the goal of designing ABPs we synthesized azido- $n$-octyl manno-epi-cyclophellitol (3, Figure 2) starting from D-ribose in 15 steps and used a copper(I)catalyzed azide-alkyne $[2+3]$ cycloaddition to ligate this to either Cy5-alkyne, biotin-alkyne, or 5'/6'-TAMRA-PEG $4^{-}$ alkyne to yield ABPs 4, 5, and 6, respectively. Detailed synthetic procedures are given in the Supporting Information.

Manno-epi-cyclophellitol Aziridine Probes React with All Human GH38 $\alpha$-Mannosidases. To determine whether human mannosidases would react with the cyclophellitol aziridine probes within complex mixtures, all five human GH38 $\alpha$-mannosidases (epididymis-specific $\alpha$-mannosidase (hEpman), hGMII, hLAM, hMan2A2, and hMan2C1) were individually cloned and expressed in HEK293T cells, and extracts thereof treated with $3 \mu \mathrm{M}$ ABP 4 in buffers with $\mathrm{pH}$ between 4 and 7.5 for $30 \mathrm{~min}$ at $37^{\circ} \mathrm{C}$. All five human GH38s could be detected by in-gel fluorescence scanning of gels containing SDS-PAGE-separated proteins with intensities greater than that seen for a mock (no transfection) control. In addition, each of the five cloned enzymes proved to react with the probe in a $\mathrm{pH}$-dependent manner. Lysates of cells transfected with the Golgi-resident hGMII and hMan2A2 and treated with 4 gave similar labeling patterns with bands at approximately $140 \mathrm{kDa}$ and maximal read-out at $\mathrm{pH}$ 5.5-6.0
(Figure 4A). Lysates of cells transfected with the lysosomal hLAM gave multiple bands with highest intensities when derived from samples treated at $\mathrm{pH} 4.0-5.5$, with molecular weights of $\sim 110,65,45$, and $30 \mathrm{kDa}$. This band pattern reflects the complex processing and maturation of hLAM in lysosomes $^{28}$ (Figure 4A) and moreover shows all the processed forms to be reactive toward ABP 4.

Lysosomal hEpman has previously been purified from HEK293 cells $^{29}$ as a $\sim 110 \mathrm{kDa}$ protein. Treatment of lysates of cells transfected with lysosomal hEpman with ABP 4 gave after SDS PAGE and gel slab fluorescence scanning the expected band at $\sim 110 \mathrm{kDa}$, accompanied by an additional, more intense band at $\sim 50 \mathrm{kDa}$, which we hypothesize represents a previously unreported proteolytic product. Both the $\sim 110$ and $50 \mathrm{kDa}$ band intensities were maximal from samples treated at $\mathrm{pH} 4.0-5.5$ (Figure 4A). Lysates of cells transfected with the cytosolic hMan2C1 gave after $\mathrm{ABP} 4$ treatment an expected band just above $100 \mathrm{kDa}$, matching what has been previously reported for hMan2C1 overexpression in HEK293 cells. ${ }^{30}$ Band intensity proved most prominent at $\mathrm{pH}$ 5.5-7.5, which is more alkaline than those witnessed for other GH38 enzymes expressed in HEK293T cells (Figure 4A). While ABPP does not allow discriminating the two Golgi GH38 $\alpha$-mannosidases due to their similar $\mathrm{pH}$ range and molecular weight, the other three enzymes are readily identifiable by $A B P$ labeling at different $\mathrm{pH}$ values (Figure 4A).

Comparative ABPP Reveals Tissue-Dependent Enzyme Activity Patterns. We next sought to establish whether 

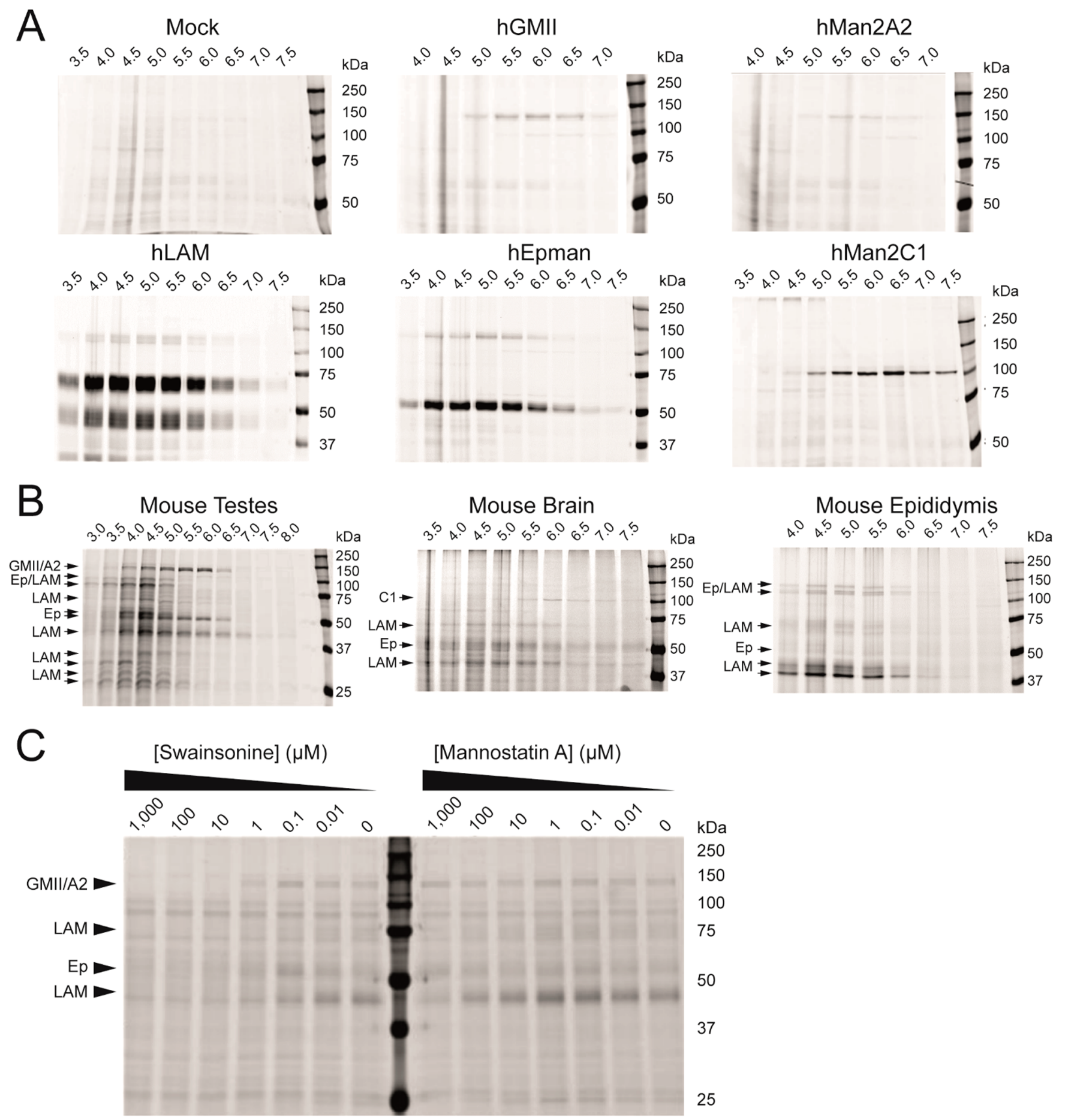

Figure 4. (A) ABPP of lysate from HEK293T cells expressing human GH38 $\alpha$-mannosidases. ABP 4 was used to show activity patterns for hGMII, hMan2A2, hLAM, hEpman, and hMan2C1 expressing cells. (B) Differential activity of GH38 $\alpha$-mannosidases in mouse tissue extracts labeled with $\mathrm{ABP}$ 4. (C) Competitive ABPP of swainsonine or mannostatin A with ABP 4 in mouse testis homogenate. Arrows indicate bands that were abolished by swainsonine preincubation. Arrows in B and C show positions of specific proteins with Man2A2, Epman, and Man2C1 abbreviated A2, Ep, and C1, respectively.

the fluorescent ABP 4 could be used to profile GH38 $\alpha$ mannosidase activity across various tissues. We focused our attention on mouse tissues as mice express homologues of all five human GH38s, hereafter referred to as mGMII, mMan2A2, mLAM, mEpman, and mMan2C1. Extracts from mouse testes, epididymis, and brain were treated with $3 \mu \mathrm{M}$ $\mathrm{ABP} 4$ for $30 \mathrm{~min}$ at $37^{\circ} \mathrm{C}$. Mouse testes and epididymis were chosen, as both Man2A2 and Epman have been implicated in spermatogenesis, $^{31,32}$ while brain tissue was chosen, as Man2C1 has previously been reported to be expressed in the brain. ${ }^{33}$ Ensuing SDS PAGE separation of the protein content and fluorescence imaging of the resultant wet gel slabs revealed concentration-dependent labeling (Figure S2) and quite distinct GH38 activity patterns (Figure 4B). Mouse mGMII and mMan2A2 proved most prominently expressed in mouse testis and less in mouse brain and epididymis. Mouse mLAM 
and mEpman in contrast are expressed by all tissues, while detectable levels of mMan2C1 were only observed in mouse brain (Figure 4B). In mouse epididymis extracts, the 65 and 45 $\mathrm{kDa}$ mLAM species seemed to have multiple forms that differ in molecular weights, in contrast to the sharp bands obtained from ABP 4-treated mouse brain extracts.

To allow identification of the GH38 mannosidases by means of chemical proteomics, treatment of mouse testis extracts with biotin-ABP $\mathbf{5}$ was performed. Samples incubated with ABP 5 at either $\mathrm{pH} 4.5$ or 6.0 were subjected to biotin affinity enrichment and tryptic digestion (both on-bead digestion and in-gel digestion), and finally LC-MS-based protein identification. While silver staining of the gel containing the affinity-enriched samples yielded few, if any, distinct bands (Figure S3), the on-bead digest protocol identified MLAM and mEpman from the sample treated with ABP 5 at $\mathrm{pH} 4.5$ and all five $\mathrm{GH} 38 \alpha$-mannosidases in the sample treated at $\mathrm{pH} 6.0$ (Table S3). No other glycosidases were detected in this manner from these samples, and full peptide mass spec results are available as Supporting Information.

Protein identity was also confirmed through a competitive ABPP (cABPP) experiment. Mouse testis extracts were preincubated with swainsonine or mannostatin A for $30 \mathrm{~min}$ at $\mathrm{pH} 4.5$ and then incubated with $3 \mu \mathrm{M} \mathrm{ABP} 4$ for $10 \mathrm{~min}$ at $37^{\circ} \mathrm{C}$. The emergence of the $140,50,45$, and $75 \mathrm{kDa}$ bands was reduced by swainsonine preincubation, and the $45 \mathrm{kDa}$ band was also competed away with $1 \mathrm{mM}$ mannostatin A (Figure 4C).

ABPP-Based Screening for GMII Inhibitors. The successful detection of GH38 $\alpha$-mannosidases and our ability to block probe modification with competitive inhibitors suggested that a high-throughput screen for inhibitors using a fluorescence polarization (FluoPol) ABPP assay would work as well. FluoPol screens offer several desirable qualities including low requirements for both probe and enzyme and the ability to screen complex enzyme mixtures. ${ }^{2,34}$ Furthermore, FluoPol ABPP enables specific targeting of competitive inhibitors; inhibitors that bind after the probe will not result in a decreased signal.

For FluoPol screening we used the TAMRA-derivatized probe 6-as opposed to the Cy5 derivative 4-as TAMRA has a longer fluorescence lifetime and thus may provide a better signal-to-noise ratio. ${ }^{35}$ The TAMRA-derivatized ABP 6 also displays time-dependent inhibition of dGMII, with an inhibition constant of $2100 \pm 300 \mathrm{~min}^{-1} \mathrm{M}^{-1}$, approximately 2.6-fold less than 2 (see Figure S1). Initial tests toward such a FluoPol ABPP assay were performed using 96-well plates with each well containing $100 \mathrm{nM}$ dGMII and $25 \mathrm{nM}$ of probe and the known inhibitors mannostatin $\mathrm{A}$ and swainsonine at various concentrations. Protein concentration was set to be in excess of the probe concentration to ensure maximum fluorescence polarization signals. Although the $\mathrm{pH}$ optimum for dGMII on chromogenic substrates has previously been reported $^{36}$ as 5.7, we observed an optimal ABP binding at $\mathrm{pH}$ 6.5. This alkaline shift is similar to what we observed for human nonlysosomal glucosylceramidase (GBA2). ${ }^{22}$ The inhibition curve for swainsonine shows reduced inhibition at nanomolar concentrations-as this is a low nanomolar inhibitor $\left(K_{\mathrm{i}}=20 \mathrm{nM}^{19}\right)$, this reflects the enzyme concentration in the assay-while the curve for mannostatin A $\left(K_{\mathrm{i}}=36 \mathrm{nM}^{37}\right)$ showed reduced inhibition in the micromolar range (see Figure 5A). Both inhibitors significantly decreased polarization of TAMRA-emitted light in a

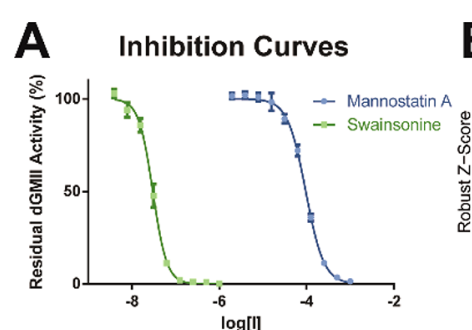

B Iminosugar Screen
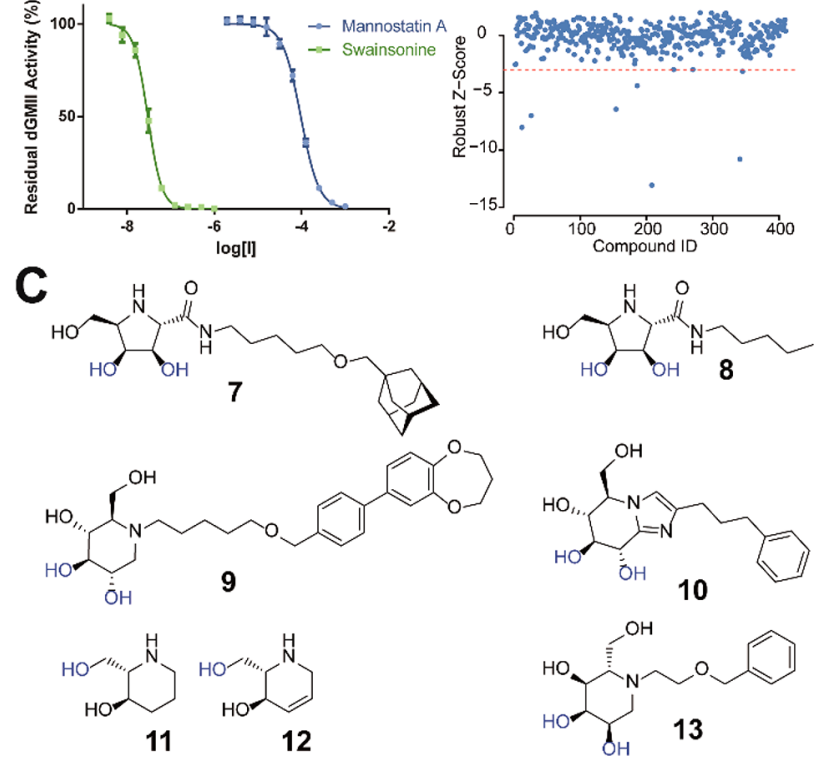

Figure 5. Screen validation, results, and identified inhibitors. (A) IC $_{50}$ curves for mannostatin $\mathrm{A}$ and swainsonine determined with the developed FluoPol assay. (B) FluoPol screening results. Red dashed line indicates a Robust $Z$-score of -3. (C) Structures of the identified inhibitors. Hydroxyls that interact with the active site zinc are shown in blue.

concentration-dependent manner, and a difference in polarized light emission of approximately $250 \mathrm{mPol}$ was achieved for the completely inhibited enzyme. The resulting $Z^{\prime}$-score for the assay was $>0.9$, indicating that even with the low concentration of $\mathrm{ABP}$ used, the assay is amenable for high-throughput screening. ${ }^{38}$

We next screened our in-house library of 358 iminosugars for dGMII inhibition. We used this continuously evolving library previously to identify, in a FluoPol ABPP manner, GBA2 inhibitors. ${ }^{22}$ The library includes, among others, pyrrolidine and piperidine iminosugars featuring a variety of configurations and $\mathrm{N}$-alkylations. Screening was performed with dGMII, which can be purified in reasonable quantities, as necessary enzyme quantities were not known a priori. However, the developed assay requires very little material $(0.58 \mathrm{nmol}$ of dGMII and $0.15 \mathrm{nmol}$ of 6 for two 384-well plates); therefore it should enable future screening of $\alpha$ mannosidases that can only be obtained in small quantities. All compounds in the iminosugar library were screened at a final concentration of $5 \mu \mathrm{M}$, and the relative loss of fluorescence polarization as a function of inhibitor was determined. A total of seven compounds produced at least a $20 \%$ reduction in activity of dGMII (robust $Z$-score $>3$ ) and were selected for further analysis (Figure 5). Two of these compounds are Dlyxo-configured pyrrolidines (7 and 8), featuring different $\mathrm{N}$ alkyl substituents. The stereochemistry of the pseudoanomeric carbon in these D-lyxo compounds is conserved and important for binding, as compounds with the opposite pseudoanomeric configuration that are also present in our library did not significantly decrease dGMII activity.

Nearly one-third of the compounds in the library are $(\mathrm{N}$ alkylated) configurational deoxynojirimycin analogues. Only one of these (9) reduced dGMII activity, which may not be surprising given the poor inhibition of the enzyme by the 
"parent" deoxymannojirimycin. ${ }^{39}$ Our FluoPol ABPP assay also identified phenylpropyl-substituted glucoimidazole (10), the only sugar-imidazole within the library, which is consistent with the previous identification of both glucoimidazole and mannoimidazole as dGMII inhibitors. ${ }^{15}$ The seven compounds identified further include 2-hydroxymethyl-3-piperidinol (11) and its 4,5-unsaturated analogue (12). The final inhibitor we identified is 1-deoxy-L-allo-nojirimycin derivative (13), which entails the first example, as far as we know, of an alloseconfigured piperidine inhibiting dGMII.

To assess the inhibitory potency of the seven identified hits, we determined $\mathrm{IC}_{50}$ values using the FluoPol assay and $K_{\mathrm{i}}$ values using the fluorogenic MU- $\alpha$-Man (Table 1, Figures S4

\section{Table 1. $K_{\mathrm{i}}$ and $\mathrm{IC}_{50}$ Values for the Identified Inhibitors}

\begin{tabular}{|c|c|c|c|}
\hline inhibitor & $\mathrm{dGMII} \mathrm{IC}_{50}(\mu \mathrm{M})^{a}$ & dGMII $K_{\mathrm{i}}(\mu \mathrm{M})^{b}$ & hGMII $K_{\mathrm{i}}(\mu \mathrm{M})^{b}$ \\
\hline 7 & $6.1 \pm 0.5$ & $8.4 \pm 0.4$ & $20 \pm 2$ \\
\hline 8 & $8.7 \pm 0.9$ & $10.8 \pm 0.8$ & $41 \pm 3$ \\
\hline 9 & $50 \pm 5$ & $140 \pm 40$ & $270 \pm 10$ \\
\hline 10 & $18 \pm 2$ & $12.5 \pm 0.8$ & $45 \pm 6$ \\
\hline 11 & $18 \pm 1$ & $58 \pm 3$ & $100 \pm 7$ \\
\hline 12 & $56 \pm 6$ & $180 \pm 10$ & $770 \pm 70$ \\
\hline 13 & $22 \pm 2$ & $39 \pm 2$ & $51 \pm 2$ \\
\hline
\end{tabular}

${ }^{a}$ Determined using the FluoPol assay. ${ }^{b}$ Determined by initial rates of MU- $\alpha$-Man hydrolysis in the presence of inhibitor.

and S5). The ranking of the inhibitors is equivalent for both $\mathrm{IC}_{50}$ and $K_{\mathrm{i}}$, apart from 9 (Table 1 ). The most potent inhibitors of dGMII proved to be the D-lyxo-configured iminosugars, with $K_{\mathrm{i}}$ values of 8 and $10.8 \mu \mathrm{M}$ for 7 and 8 , respectively. The hydroxylated pyrrolidine core of these inhibitors has previously been reported, ${ }^{17}$ and a derivatized version (Figure 1B) has been shown to be a nanomolar inhibitor of hGMII. ${ }^{3}$ The tolerance of dGMII toward the bulky adamantane-1-yl-methoxy ether warrants further studies to investigate whether larger substituents would impart increased inhibition and/or selectivity. Phenylpropyl-substituted glucoimidazole (10) also inhibited dGMII in the low micromolar range $\left(K_{\mathrm{i}}: 12.5 \mu \mathrm{M}\right)$. This corresponds very well with the inhibition observed by Kuntz et al. for unsubstituted glucoimidazole $\left(K_{\mathrm{i}}: 13 \mu \mathrm{M}\right.$ with dGMII). ${ }^{15}$ The nearly equivalent inhibition of the substituted glucoimidazole raises the possibility that diversification of the substitution may enable the future generation of selective inhibitors. The remaining four identified inhibitors all had $K_{\mathrm{i}}$ values of $<200$ $\mu \mathrm{M}$ (Table 1) for dGMII despite little literature precedence for their inhibition.

Ultimately, the therapeutic potential of discovered inhibitors will rely on their inhibition of the human Golgi $\alpha$-mannosidase (hGMII). We therefore tested the inhibition of recombinant hGMII with the identified inhibitors, under the same conditions used for dGMII. All seven identified compounds had inhibition constants in the micromolar range, though all had higher inhibition constants for the human enzyme compared to dGMII (Table 1). Nonetheless, five of the seven identified inhibitors have $K_{\mathrm{i}}$ values of $100 \mu \mathrm{M}$ or less for hGMII.

We next performed $c A B P P$ with the inhibitors 8 and $\mathbf{1 0}$, representing the two best classes of inhibitors discovered to assess the inhibitor specificity. Mouse testis extracts were preincubated with 8 or $\mathbf{1 0}$ for $30 \mathrm{~min}$ at $\mathrm{pH} 6.5$ and then incubated with $3 \mu \mathrm{M}$ ABP 4 for $15 \mathrm{~min}$ at $37^{\circ} \mathrm{C}$. The 140,50 , and $45 \mathrm{kDa}$ bands (corresponding to $\mathrm{mGMII} / \mathrm{mMan} 2 \mathrm{~A} 2$, mEpman, and mLAM) were reduced by preincubation with 8 (Figure S9). Preincubation with $\mathbf{1 0}$ resulted in the loss of the $45 \mathrm{kDa}$ band (mLAM) in the presence of $200 \mu \mathrm{M}$ inhibitor (Figure S9).

Crystallography of dGMII with Inhibitors. To further understand the action of the $\alpha$-mannosidase inhibitors, we produced structures of dGMII, in complex with the inhibitors by soaking dGMII crystals with solutions containing 7-13. The electron density for the bound inhibitors allowed the modeling of all inhibitors. Only the alkyl tails attached to 9, 10, and $\mathbf{1 3}$ had poor density and were accordingly only partially modeled. Unusual among GHs, GMII contains an active-site zinc, which coordinates substrate 2- and 3-hydroxyls. ${ }^{21}$ All seven inhibitors bind to the active site of dGMII and coordinate to the active site zinc (Figures 6A, S6, and S7).

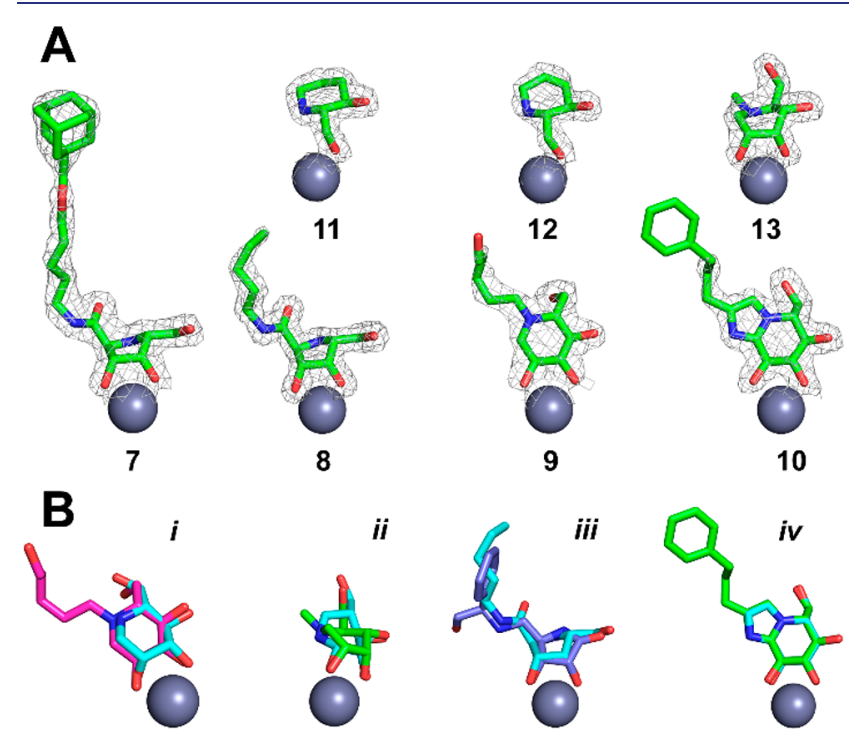

Figure 6. Configuration of bound dGMII inhibitors and structural comparisons to known inhibitors. (A) Inhibitor density of the identified inhibitors. The active site zinc is shown as a gray sphere, and $2 F_{\mathrm{o}}-F_{\mathrm{c}}$ maps are contoured at $1.5 \sigma$, except for 5 , which is contoured at $1 \sigma$. (B) Comparison of the binding of discovered inhibitors to the binding of previously identified inhibitors. (i) Overlay of 9 and deoxymannojirimycin (PDB: 1HXK). (ii) Overlay of 13 and noeuromycin (PDB: 2ALW). (iii) Overlay of 8 and a derivatized pyrrolidinone (PDB: 3DDF). (iv) Overlay of $\mathbf{1 0}$ and glucoimidazole (PDB: 3D4Z).

Both D-lyxo-configured iminosugars are bound in an ${ }^{\mathrm{N}} T_{4}$ conformation with 2- and 3-hydroxyls coordinating the active site zinc. The alkyl groups of both 7 and 8 are projected toward the "+" sugar binding sites. The observed ${ }^{\mathrm{N}} T_{4}$ conformation is consistent with the pyrrolidine inhibitor $\left(K_{\mathrm{i}}\right.$ : $67 \mu \mathrm{M}$, PDB: $2 \mathrm{~F} 18)$ identified by Englebienne et al. ${ }^{40}$ This inhibitor lacks the 5-hydroxymethyl substituent present in 7 and 8, which interacts with Asp472 and Tyr727 in the structures presented here. Fiaux et al. ${ }^{15}$ also presented two dGMII structures with pyrrolidone-based inhibitors resembling 7 and $8\left(K_{\mathrm{i}}: 22\right.$ and $1 \mu \mathrm{M}$, PDB: 3DDF and 3DDG). These pyrrolidones, due to the planarity imposed by the lactam, are present in a ${ }^{3} T_{2}$ conformation (numbering based on the D-lyxo analogue) and can interact with Asp472 and Tyr727 through the lactam carbonyl. The amide carbonyl of 7 and 8 is also within hydrogen-bonding distance of an active site water, an 
interaction that is missing in PDB structures 2F18 and 3DDG. An amide is also present in the derivatized pyrrolidine (Figure 1) identified by Cheng et al.; ${ }^{17}$ however it is positioned one carbon away from the iminosugar ring, and therefore may not be able to participate in this hydrogen bond. Incorporation of a (4-fluorophenyl)acetamide, described by Cheng et al., ${ }^{17}$ in place of the alkyl substituent of 7 may indeed increase potency of this inhibitor.

Investigation of the alkylated glucoimidazole inhibitor revealed a binding mode similar to that previously seen for glucoimidazole (Figure 6B). Both inhibitors are present in a ${ }^{2} \mathrm{H}_{3} / E_{3}$ conformation and coordinate the active site zinc with the 2- and 3-hydroxyls. The electron density of the 7phenylpropyl could only be assigned to two of the alkyl carbons (Figure 6A), suggesting conformational flexibility of the alkyl group. Further investigation of derivatives at the 7position of glucoimidazole or mannoimidazole-a better inhibitor-could enable the creation of more potent and selective inhibitors.

The two structures containing the hydroxylated piperidines 11 and 12 bind to dGMII in a very similar manner (Figure S7). Unlike all other known inhibitors of dGMII that coordinate zinc with two hydroxyls, $\mathbf{1 1}$ and $\mathbf{1 2}$ coordinate the active site zinc with a single hydroxyl. This results in a five-coordinate complex, as opposed to the six-coordinate zinc that is typically observed. The hydroxyl that coordinates zinc is also within hydrogen bond distance of Asp472 and the nucleophile Asp204. The other hydroxyl is appropriately positioned to form hydrogen bonds with Asp472 and Tyr727. The ring nitrogen for both 11 and $\mathbf{1 2}$ also interacts closely (2.9 $\AA$ ) with the nucleophile Asp204. Although the polar interactions for 11 and 12 appear very similar, the piperidine ring conformations are different $\left({ }^{1} C_{4}\right.$ for 11 and ${ }^{5} H_{\mathrm{N}}$ for 12) due to the presence of an unsaturated bond in $\mathbf{1 2}$.

The binding of 13 was also atypical for dGMII inhibitors (Figures 6 and S6). Two pyranose iminosugars, namely, noeuromycin ${ }^{41}$ ( $\mathrm{IC}_{50}: 20 \mu \mathrm{M}, \mathrm{PDB}: 2 \mathrm{ALW}$ ) and deoxymannojirimycin $^{39}$ (DMJ, $\mathrm{IC}_{50}$ : $400 \mu \mathrm{M}, \mathrm{PDB}: 1 \mathrm{HXK}$ ), have previously been shown to bind dGMII. When compared to these two inhibitors, 13 appears to form interactions with dGMII similar to noeuromycin (Figures 6B, S6), with 2- and 3$\mathrm{OH}$ coordinating with the active site zinc, the 4-OH hydrogen bonding to Asp472 and Tyr727, the 6-OH hydrogen bonding to $A r g 876$, and the ring nitrogen positioned within $3 \AA$ of the nucleophile carbonyl (Asp204). However, the differing C4 and C5 epimers of 13 necessitate a $B_{1,4}$ conformation as opposed to the ${ }^{1} S_{5}$ conformation of noeuromycin. The deoxynojirimycin derivative 9 conversely appears to bind to dGMII in a manner more akin to DMJ. In the structure of both 9 and DMJ, the ring nitrogen is no longer in position to form a hydrogen bond with the active site nucleophile, but instead hydrogen bonds to an active site water. This loss of the interaction between the ring nitrogen and the active site nucleophile is likely the cause of the decreased relative inhibition of DMJ and 9 when compared to $\mathbf{1 3}$ and noeuromycin. One final difference seen in the structure of 9 binding to dGMII is the positioning of the 6$\mathrm{OH}$. The structure with 9 shows the 6-OH hydrogen bonding to the $\omega$-nitrogen of Arg288, instead of the carbonyl of Arg876, which is observed for all other pyranose iminosugars.

\section{CONCLUSIONS}

Manno-epi-cyclophellitols have proven to be powerful tools for the study of GH38 $\alpha$-mannosidases and the identification of new inhibitors. The epoxide and aziridine analogues and fluorescent derivatives are covalent time-dependent inhibitors. X-ray crystallography has revealed the ${ }^{1} S_{5}$ conformation of these cyclophellitols, which is consistent with theoretical predictions for the glycosyl-enzyme intermediate. Fluorescent derivatives have enabled the profiling of tissue-dependent GH38 activity and a previously unseen proteolyzed form of hEpman. Additionally, a FluoPol-ABPP screen with excellent characteristics and low substrate and enzyme requirements was developed using the fluorescently tagged manno-epi-cyclophellitol aziridine. This screen revealed seven new inhibitors of both dGMII and hGMII, including D-lyxo-configured pyrrolidines, deoxynojirimycins, hydroxylated piperidines, and an alkylated glucoimidazole. X-ray crystallography of these compounds bound to dGMII revealed both new binding modes and potential sites for inhibitor diversification. This work sets the stage for future development of selective GMII inhibitors. Furthermore, the low material cost in terms of both enzyme and substrate of the screen developed herein will enable the screening of $\alpha$-mannosidases, which may only be expressed in small quantities. This in turn should enable the screening for inhibitors of all human $\alpha$-mannosidases.

\section{EXPERIMENTAL SECTION}

Methodology for protein expression, purification, kinetic analysis, inhibitor-library screening, the conditions for crystal growth and inhibitor soaking, the methods for data collection and structure refinement, and the synthesis of manno-epi-cyclophellitol inhibitors and probes are reported in the Supporting Information. The atomic coordinates and structure factors have been deposited in the Protein Data Bank. (PDB ID codes: 6RQZ, 6RRH, 6RRJ, 6RRN, 6RRW, 6RRU, 6RRX, 6RRY, and 6RS0).

\section{ASSOCIATED CONTENT}

\section{(s) Supporting Information}

The Supporting Information is available free of charge at https://pubs.acs.org/doi/10.1021/jacs.0c03880.

Additional information (PDF)

\section{AUTHOR INFORMATION}

\section{Corresponding Authors}

Herman S. Overkleeft - Leiden Institute of Chemistry, Leiden University, 2333 CC Leiden, The Netherlands; 이이.org/ 0000-0001-6976-7005; Email: h.s.overkleeft@

chem.leidenuniv.nl

Gideon J. Davies - Structural Biology Laboratory, Department of Chemistry, The University of York, York YO10 5DD, United Kingdom; 10 orcid.org/0000-0002-7343-776X; Email: gideon.davies@york.ac.uk

\section{Authors}

Zachary Armstrong - Structural Biology Laboratory, Department of Chemistry, The University of York, York YO10 5DD, United Kingdom; () orcid.org/0000-0002-4086-2946

Chi-Lin Kuo - Leiden Institute of Chemistry, Leiden University,

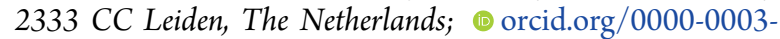
3748-5008

Daniël Lahav - Leiden Institute of Chemistry, Leiden University, 2333 CC Leiden, The Netherlands

Bing Liu - Leiden Institute of Chemistry, Leiden University, 2333 CC Leiden, The Netherlands 
Rachel Johnson - Structural Biology Laboratory, Department of Chemistry, The University of York, York YO10 5DD, United Kingdom

Thomas J. M. Beenakker - Leiden Institute of Chemistry, Leiden University, 2333 CC Leiden, The Netherlands

Casper de Boer - Leiden Institute of Chemistry, Leiden University, 2333 CC Leiden, The Netherlands; (1) orcid.org/ 0000-0002-0869-6060

Chung-Sing Wong - Leiden Institute of Chemistry, Leiden University, 2333 CC Leiden, The Netherlands

Erwin R. van Rijssel - Leiden Institute of Chemistry, Leiden University, 2333 CC Leiden, The Netherlands

Marjoke F. Debets - Leiden Institute of Chemistry, Leiden University, 2333 CC Leiden, The Netherlands

Bogdan I. Florea - Leiden Institute of Chemistry, Leiden University, 2333 CC Leiden, The Netherlands; (1) orcid.org/ 0000-0001-7114-2266

Colin Hissink - Leiden Institute of Chemistry, Leiden University, 2333 CC Leiden, The Netherlands

Rolf G. Boot - Leiden Institute of Chemistry, Leiden University, 2333 CC Leiden, The Netherlands

Paul P. Geurink - Oncode Institute \& Department of Cell and Chemical Biology, Leiden University Medical Centre, 2333 ZC Leiden, The Netherlands; 10 orcid.org/0000-0003-1849-1111

${ }^{\perp}$ Huib Ovaa - Oncode Institute \& Department of Cell and Chemical Biology, Leiden University Medical Centre, 2333 ZC Leiden, The Netherlands; 이이.orid/0000-0002-0068-054X

Mario van der Stelt - Leiden Institute of Chemistry, Leiden University, 2333 CC Leiden, The Netherlands; (1) orcid.org/ 0000-0002-1029-5717

Gijsbert M. van der Marel - Leiden Institute of Chemistry, Leiden University, 2333 CC Leiden, The Netherlands; (1) orcid.org/0000-0003-3531-2138

Jeroen D. C. Codée - Leiden Institute of Chemistry, Leiden University, 2333 CC Leiden, The Netherlands

Johannes M. F. G. Aerts - Leiden Institute of Chemistry, Leiden University, 2333 CC Leiden, The Netherlands

Liang Wu - Structural Biology Laboratory, Department of Chemistry, The University of York, York YO10 5DD, United

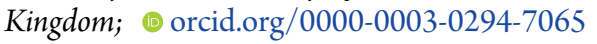

Complete contact information is available at: https://pubs.acs.org/10.1021/jacs.0c03880

\section{Notes}

The authors declare no competing financial interest.

${ }^{\perp}$ Huib Ovaa passed away on May 19, 2020.

\section{ACKNOWLEDGMENTS}

We thank The Netherlands Organization for Scientific Research (NWO; TOP grant to H.S.O.), the European Research Council (ERC-2011-AdG-290836 "Chembiosphing” to H.S.O. and ERC-2012-AdG-32294 "Glycopoise" to G.J.D.), and the Biotechnology and Biological Sciences Research Council (BBSRC; BB/R001162/1 and BB/M011151/1 grants to G.J.D.). G.J.D. thanks the Royal Society for the Ken Murray Research Professorship. We thank the Diamond Light Source for access to beamlines i03 and i04 (proposal number $\mathrm{mx} 18598$ ) that contributed to the results presented here. The authors dedicate this paper to the late Huib Ovaa (19732020).

\section{REFERENCES}

(1) Lombard, V.; Golaconda Ramulu, H.; Drula, E.; Coutinho, P. M.; Henrissat, B. The carbohydrate-active enzymes database (CAZy) in 2013. Nucleic Acids Res. 2014, 42 (Database issue), D490-D495.

(2) Shah, N.; Kuntz, D. A.; Rose, D. R. Golgi $\alpha$-mannosidase II cleaves two sugars sequentially in the same catalytic site. Proc. Natl. Acad. Sci. U. S. A. 2008, 105 (28), 9570-9575.

(3) Dennis, J. W.; Laferte, S.; Waghorne, C.; Breitman, M. L.; Kerbel, R. S. $\beta 1-6$ branching of Asn-linked oligosaccharides is directly associated with metastasis. Science 1987, 236 (4801), 582585.

(4) Pierce, M.; Buckhaults, P.; Chen, L.; Fregien, N. Regulation of $\mathrm{N}$-acetylglucosaminyltransferase $\mathrm{V}$ and Asn-linked oligosaccharide $\beta(1,6)$ branching by a growth factor signaling pathway and effects on cell adhesion and metastatic potential. Glycoconjugate J. 1997, 14 (5), 623-630.

(5) Colegate, S. M.; Dorling, P. R.; Huxtable, C. R. A spectroscopic investigation of swainsonine: an $\alpha$-mannosidase inhibitor isolated from Swainsona canescens. Aust. J. Chem. 1979, 32 (10), 2257-2264.

(6) Mohla, S.; Humphries, M. J.; White, S. L.; Matsumoto, K.; Newton, S. A.; Sampson, C. C.; Bowen, D.; Olden, K. Swainsonine: a new antineoplastic immunomodulator. J. Natl. Med. Assoc. 1989, 81 (10), 1049-1056.

(7) Dennis, J. W.; Koch, K.; Beckner, D. Inhibition of human HT29 colon carcinoma growth in vitro and in vivo by swainsonine and human interferon- $\alpha 2$ 2. Journal of the National Cancer Institute 1989, 81 (13), 1028-1033.

(8) Dennis, J. W.; Koch, K.; Yousefi, S.; VanderElst, I. Growth inhibition of human melanoma tumor xenografts in athymic nude mice by swainsonine. Cancer Res. 1990, 50 (6), 1867-1872.

(9) Cenci di Bello, I.; Dorling, P.; Winchester, B. The storage products in genetic and swainsonine-induced human mannosidosis. Biochem. J. 1983, 215 (3), 693-696.

(10) Huxtable, C. R.; Dorling, P. R. Animal model of human disease. Mannosidosis. Swainsonine-induced mannosidosis. Am. J. Pathol. 1982, 107 (1), 124-126.

(11) Kuntz, D. A.; Nakayama, S.; Shea, K.; Hori, H.; Uto, Y.; Nagasawa, H.; Rose, D. R. Structural Investigation of the Binding of 5 -Substituted Swainsonine Analogues to Golgi $\alpha$-Mannosidase II. ChemBioChem 2010, 11 (5), 673-680.

(12) Aoyagi, T.; Yamamoto, T.; Kojiri, K.; Morishima, H.; Nagai, M.; Hamada, M.; Takeuchi, T.; Umezawa, H. Mannostatins A and B: new inhibitors of $\alpha$-D-mannosidase, produced by Streptoverticillium verticillus var. quintum ME3-AG3: taxonomy, production, isolation, physico-chemical properties and biological activities. J. Antibiot. 1989, 42 (6), 883-889.

(13) Li, B.; Kawatkar, S. P.; George, S.; Strachan, H.; Woods, R. J.; Siriwardena, A.; Moremen, K. W.; Boons, G.-J. Inhibition of Golgi Mannosidase II with Mannostatin A Analogues: Synthesis, Biological Evaluation, and Structure-Activity Relationship Studies. ChemBioChem 2004, 5 (9), 1220-1227.

(14) Popowycz, F.; Gerber-Lemaire, S.; Demange, R.; RodriguezGarcía, E.; Asenjo, A. T. C.; Robina, I.; Vogel, P. Derivatives of (2R,3R,4S)-2-Aminomethylpyrrolidine-3,4-diol are selective $\alpha$-Mannosidase inhibitors. Bioorg. Med. Chem. Lett. 2001, 11 (18), 24892493.

(15) Fiaux, H.; Kuntz, D. A.; Hoffman, D.; Janzer, R. C.; GerberLemaire, S.; Rose, D. R.; Juillerat-Jeanneret, L. Functionalized pyrrolidine inhibitors of human type II $\alpha$-mannosidases as anti-cancer agents: Optimizing the fit to the active site. Bioorg. Med. Chem. 2008, 16 (15), 7337-7346.

(16) Fiaux, H.; Popowycz, F.; Favre, S.; Schütz, C.; Vogel, P.; Gerber-Lemaire, S.; Juillerat-Jeanneret, L. Functionalized Pyrrolidines Inhibit $\alpha$-Mannosidase Activity and Growth of Human Glioblastoma and Melanoma Cells. J. Med. Chem. 2005, 48 (13), 4237-4246.

(17) Cheng, T.-J. R.; Chan, T.-H.; Tsou, E.-L.; Chang, S.-Y.; Yun, W.-Y.; Yang, P.-J.; Wu, Y.-T.; Cheng, W.-C. From Natural ProductInspired Pyrrolidine Scaffolds to the Development of New Human 
Golgi $\alpha$-Mannosidase II Inhibitors. Chem. - Asian J. 2013, 8 (11), $2600-2604$.

(18) Gerber-Lemaire, S.; Popowycz, F.; Rodriguez-Garcia, E.; Asenjo, A. T.; Robina, I.; Vogel, P. An efficient combinatorial method for the discovery of glycosidase inhibitors. ChemBioChem 2002, 3 (5), 466-470.

(19) Kuntz, D. A.; Tarling, C. A.; Withers, S. G.; Rose, D. R. Structural Analysis of Golgi $\alpha$-Mannosidase II Inhibitors Identified from a Focused Glycosidase Inhibitor Screen. Biochemistry 2008, 47 (38), 10058-10068.

(20) Wu, L. A.; Armstrong, Z.; Schröder, S. P.; de Boer, C.; Artola, M.; Aerts, J. M. F. G.; Overkleeft, H. S.; Davies, G. An overview of activity-based probes for glycosidases. Curr. Opin. Chem. Biol. 2019, $53,25-36$.

(21) Numao, S.; Kuntz, D. A.; Withers, S. G.; Rose, D. R. Insights into the Mechanism of Drosophila melanogaster Golgi $\alpha$-Mannosidase II through the Structural Analysis of Covalent Reaction Intermediates. J. Biol. Chem. 2003, 278 (48), 48074-48083.

(22) Lahav, D.; Liu, B.; van den Berg, R. J. B. H. N.; van den Nieuwendijk, A. M. C. H.; Wennekes, T.; Ghisaidoobe, A. T.; Breen, I.; Ferraz, M. J.; Kuo, C.-L.; Wu, L.; Geurink, P. P.; Ovaa, H.; van der Marel, G. A.; van der Stelt, M.; Boot, R. G.; Davies, G. J.; Aerts, J. M. F. G.; Overkleeft, H. S. A Fluorescence Polarization Activity-Based Protein Profiling Assay in the Discovery of Potent, Selective Inhibitors for Human Nonlysosomal Glucosylceramidase. J. Am. Chem. Soc. 2017, 139 (40), 14192-14197.

(23) Schröder, S. P.; de Boer, C.; McGregor, N. G. S.; Rowland, R. J.; Moroz, O.; Blagova, E.; Reijngoud, J.; Arentshorst, M.; Osborn, D.; Morant, M. D.; Abbate, E.; Stringer, M. A.; Krogh, K. B. R. M.; Raich, L.; Rovira, C.; Berrin, J.-G.; van Wezel, G. P.; Ram, A. F. J.; Florea, B. I.; van der Marel, G. A.; Codée, J. D. C.; Wilson, K. S.; Wu, L.; Davies, G. J.; Overkleeft, H. S. Dynamic and Functional Profiling of XylanDegrading Enzymes in Aspergillus Secretomes Using Activity-Based Probes. ACS Cent. Sci. 2019, 5 (6), 1067-1078.

(24) Gloster, T. M.; Madsen, R.; Davies, G. J. Structural basis for cyclophellitol inhibition of a $\beta$-glucosidase. Org. Biomol. Chem. 2007, 5 (3), 444-446.

(25) Wu, L.; Jiang, J.; Jin, Y.; Kallemeijn, W. W.; Kuo, C.-L.; Artola, M.; Dai, W.; van Elk, C.; van Eijk, M.; van der Marel, G. A.; Codée, J. D. C.; Florea, B. I.; Aerts, J. M. F. G.; Overkleeft, H. S.; Davies, G. J. Activity-based probes for functional interrogation of retaining $\beta$ glucuronidases. Nat. Chem. Biol. 2017, 13 (8), 867-873.

(26) Artola, M.; Wu, L.; Ferraz, M. J.; Kuo, C.-L.; Raich, L.; Breen, I. Z.; Offen, W. A.; Codée, J. D. C.; van der Marel, G. A.; Rovira, C.; Aerts, J. M. F. G.; Davies, G. J.; Overkleeft, H. S. 1,6-Cyclophellitol Cyclosulfates: A New Class of Irreversible Glycosidase Inhibitor. ACS Cent. Sci. 2017, 3 (7), 784-793.

(27) Petersen, L.; Ardèvol, A.; Rovira, C.; Reilly, P. J. Molecular Mechanism of the Glycosylation Step Catalyzed by Golgi $\alpha$ Mannosidase II: A QM/MM Metadynamics Investigation. J. Am. Chem. Soc. 2010, 132 (24), 8291-8300.

(28) Pohlmann, R.; Hasilik, A.; Cheng, S.; Pemble, S.; Winchester, B.; von Figura, K. Synthesis of lysosomal $\alpha$-mannosidase in normal and mannosidosis fibroblasts. Biochem. Biophys. Res. Commun. 1983, 115 (3), 1083-1089.

(29) Park, C.; Meng, L.; Stanton, L. H.; Collins, R. E.; Mast, S. W.; Yi, X.; Strachan, H.; Moremen, K. W. Characterization of a Human Core-specific Lysosomal $\alpha 1,6-$ Mannosidase Involved in N-Glycan Catabolism. J. Biol. Chem. 2005, 280 (44), 37204-37216.

(30) Suzuki, T.; Hara, I.; Nakano, M.; Shigeta, M.; Nakagawa, T.; Kondo, A.; Funakoshi, Y.; Taniguchi, N. Man2C1, an $\alpha$-mannosidase, is involved in the trimming of free oligosaccharides in the cytosol. Biochem. J. 2006, 400 (1), 33-41.

(31) Fukuda, M. N.; Akama, T. O. In vivo role of $\alpha$-mannosidase IIx: ineffective spermatogenesis resulting from targeted disruption of the Man2a 2 in the mouse. Biochim. Biophys. Acta, Gen. Subj. 2002, 1573 (3), 382-387.

(32) Hiramoto, S.; Tamba, M.; Kiuchi, S.; Jin, Y.-Z.; Bannai, S.; Sugita, Y.; Dacheux, F.; Dacheux, J.-L.; Yoshida, M.; Okamura, N.
Stage-Specific Expression of a Mouse Homologue of the Porcine 135 $\mathrm{kDa} \alpha$-D-Mannosidase (MAN2B2) in Type A Spermatogonia. Biochem. Biophys. Res. Commun. 1997, 241 (2), 439-445.

(33) Costanzi, E.; Balducci, C.; Cacan, R.; Duvet, S.; Orlacchio, A.; Beccari, T. Cloning and expression of mouse cytosolic $\alpha$-mannosidase (Man2c1). Biochim. Biophys. Acta, Gen. Subj. 2006, 1760 (10), 15801586.

(34) Bachovchin, D. A.; Brown, S. J.; Rosen, H.; Cravatt, B. F. Identification of selective inhibitors of uncharacterized enzymes by high-throughput screening with fluorescent activity-based probes. Nat. Biotechnol. 2009, 27 (4), 387-394.

(35) Lea, W. A.; Simeonov, A. Fluorescence polarization assays in small molecule screening. Expert Opin. Drug Discovery 2011, 6 (1), $17-32$.

(36) Rabouille, C.; Kuntz, D. A.; Lockyer, A.; Watson, R.; Signorelli, T.; Rose, D. R.; van den Heuvel, M.; Roberts, D. B. The Drosophila GMII gene encodes a Golgi $\alpha$-mannosidase II. J. Cell Sci. 1999, 112, 3319-3330.

(37) Kuntz, D. A.; Zhong, W.; Guo, J.; Rose, D. R.; Boons, G.-J. The Molecular Basis of Inhibition of Golgi $\alpha$-Mannosidase II by Mannostatin A. ChemBioChem 2009, 10 (2), 268-277.

(38) Zhang, J. H. A Simple Statistical Parameter for Use in Evaluation and Validation of High Throughput Screening Assays. J. Biomol. Screening 1999, 4 (2), 67-73.

(39) van den Elsen, J. M. H. Structure of Golgi $\alpha$-mannosidase II: a target for inhibition of growth and metastasis of cancer cells. EMBO Journal 2001, 20 (12), 3008-3017.

(40) Englebienne, P.; Fiaux, H.; Kuntz, D. A.; Corbeil, C. R.; Gerber-Lemaire, S.; Rose, D. R.; Moitessier, N. Evaluation of docking programs for predicting binding of Golgi $\alpha$-mannosidase II inhibitors: A comparison with crystallography. Proteins: Struct., Funct., Genet. 2007, 69 (1), 160-176.

(41) Kuntz, D. A.; Liu, H.; Bols, M.; Rose, D. R. The role of the active site $\mathrm{Zn}$ in the catalytic mechanism of the GH38 Golgi $\alpha$ mannosidase II: Implications from noeuromycin inhibition. Biocatal. Biotransform. 2006, 24 (1-2), 55-61. 